\title{
75. A Study of the Enzyme Action by Thermal Analysis of Reaction Velocity. (I)
}

\section{The Action of Saccharase.}

\author{
By Shinkichi HoRIBA and Takekazu KosakI. \\ Chemical Institute, Kyoto Imperial University. \\ (Comm. by Y. OSAKA, M.I.A., June 12, 1935.)
}

The measurement of the chemical reaction velocity has hitherto been made either by means of chemical analysis or through the change in the physical nature of reaction systems. Horiba succeeded in deducing the reaction velocity by observing the change of heat evolved in the course of reaction. This method he named a "thermal analysis of reaction velocity," which has satisfactorily been applied to the various kinds of reaction velocity. The method can be presented by such a simple equation as this:

$$
\frac{d T}{d t}-\frac{d T^{\prime}}{d t}=\frac{Q}{W} \frac{d x}{d t}=K \frac{d x}{d t},
$$

where $\frac{d T}{d t}$ shows the observed value of the temperature change in a reaction system; $\frac{d T^{\prime}}{d t}$ the cooling velocity due to the temperature difference between a reaction system and its surroundings; $\frac{d T}{d t}-\frac{d T^{\prime}}{d t}$ the velocity of heating corrected for cooling; $Q$ the reaction heat; $W$ the heat capacity of the system; and $\frac{d x}{d t}$ the reaction velocity.

If we have observed the cooling velocity $\begin{gathered}d T^{\prime} \\ d t\end{gathered}$ particular to any reaction vessel, the value of reaction velocity $\frac{d x}{d t}$ can be easily acquired through the observation of $\frac{d T}{d t}$. Nevertheless, in a reaction in gaseous systems it is hard to make the observation of $\frac{d T}{d t}$. Therefore, under the assumption that the Ideal Gas Law should be applicable to the very case, $\frac{d x}{d t}$ may be easily calculated from the observation of $\frac{d p}{d t}$. In this case it is possible that the value of entirely rapid $\frac{d x}{d t}$ is observed. This enables us to make a considerably profound research concerning the velocity of primary reaction before it reaches the stationary state of the reaction in such cases as photochemical union of chlorine and hydrogen, ${ }^{1)}$ photochemical formation of phosgen, ${ }^{2)}$ the ex-

1) Horiba, S. and Ichikawa, T.: The Sexagint (Collection of Papers dedicated to Prof. Osaka) (1927) p. 73 ; Tamura, M., Rev. Phys. Chem. Japan, 8 (1934) p. 59.

2) Tamura, M., Ibid., 7 (1933) p. 49. 
plosion of a mixture of oxygen and hydrogen, ${ }^{1)}$ etc. Thus the method presents interesting materials for the study of reaction mechanism. In a reaction in liquid systems the direct observation of $\frac{d T}{d t}$ is possible, but we find it very difficult to observe such a high reaction velocity as that gained from $\frac{d p}{d t}$ in gaseous reactions. Nevertheless, there are some cases in the study of the earlier period of a reaction where our method is far more advantageous than any other ordinary methods. As one of those evidences we will elucidate the results of our research of the action of saccharase by means of thermal analysis.

The action of saccharase has long been a subject of research, and many valuable results have been attained. All of them, however, are what have been obtained by the observation of reduction power or degree of rotation. Accordingly, the reaction in its earlier stage is not fully explained yet. As for the reaction in its later stage it has been regarded as a first order reaction, and has been supposed to be one of homogeneous reactions. The experimental results, however, deviate a little; this has given rise to various empirical formulae, none of which are sufficient to explain the deviation.

First calculating the inversion heat of saccharose by integrating (1), we got 4.1 Cal as an average of well agreeing value. Then we observed the relation between $\frac{d x}{d t}$ and $t$ through the relation between $\frac{d T}{d t}$ and $t$. From these relations, putting $k_{m}$ thus:

$$
k_{m}=\frac{\log \left\{\left(\frac{d x}{d t}\right)_{t_{1}} /\left(\frac{d x}{d t}\right)_{t_{2}}\right\}}{0.4343\left(t_{2}-t_{1}\right)}
$$

we have obtained the relation between $k_{m}$ and $\frac{d x}{d t}$. Our analytical results prove that the inversion of saccharose by saccharase consists of the following two stages, which is not yet noticed in all experimental researches hitherto made.

(i) The earlier stage: here the $\frac{d x}{d t}-t$ curve becomes lineal. The empirical formula of reaction velocity is as follows:

$$
\frac{d x}{d t}=k_{1} \sqrt{a_{1}+x}
$$

where $k_{1}$ and $a_{1}$ are constants.

(2) The later stage: here the $\frac{d x}{d t}-k_{m}$ curve becomes lineal, and the empirical formula is as follows:

$$
\frac{d x}{d t}=b_{1} \frac{a-x}{1+c(a-x)}
$$

1) Jono, W., Rev. Phys. Chem. Japan, 9 (1935) p. 1. 
where $b_{1}$ and $c$ are constants; $a$ is the initial concentration of saccharose.

Various explanations could be made for such an experimental result. Dejecting the ordinary idea that it is a homogeneous reaction, we considered it as due to the contact-catalytic action of the colloidal system of saccharase; we derived a reaction velocity formula from the following mechanism as a heterogeneous system. And this derivation could be attained thus: the surface of enzyme is a kind of homogeneous adsorption surface as Langmuir proposed; the adsorption coefficient of saccharose is far greater than that of water; so in the earlier stage of the reaction the surface area adsorbed by saccharose is large. Accordingly, the reaction velocity relates principally to the adsorbed water. Here it is assumed that the adsorbed water dissociates into ions. As the reaction proceeds, however, the area adsorbed by saccharose becomes smaller, and the reaction velocity should depend on that adsorbed surface area. From these assumptions we derived the following formulae:

$$
\frac{d x}{d t}=k \cdot S \sqrt{\cdot \frac{b_{H_{2} O} C_{H_{2} O} O}{b_{s}}} \quad \sqrt{\left\{\left(\frac{1}{b_{s}}-a\right)+x\right\}}
$$

for the earlier stage, and

$$
\frac{d x}{d t}=k^{\prime} . S \cdot b_{s} \frac{(a-x)}{1+b_{s}(a-x)}
$$

for the later stage, where $k$ and $k^{\prime}$ are velocity constants; $b_{H_{3} O}$ and $b_{s}$ adsorption coefficients of water and saccharose respectively; $S$ the whole surface of enzyme; $C_{H_{2} O}$ the concentration of water.

These theoretical formulae coincide with the empirical ones (2) and (3) respectively, and the relation between the value of the constants$k_{1}, a_{1}, b_{1}$ and $c$ - and the experimental conditions such as the concentration of saccharose, the quantity of enzyme, the concentration of hydrogen ion satisfies the relation required by the theoretical formulae quite well.

Thus we conclude that our study of the action of saccharase as contact-catalytic has opened a new field in the research of enzymes. 